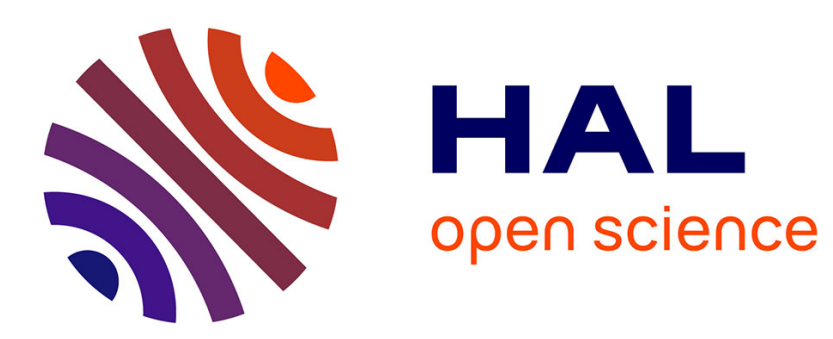

\title{
Evaporation-driven dewetting of a liquid film
}

\author{
L. Fourgeaud, E. Ercolani, J. Duplat, P. Gully, Vadim S. Nikolayev
}

\section{To cite this version:}

L. Fourgeaud, E. Ercolani, J. Duplat, P. Gully, Vadim S. Nikolayev. Evaporation-driven dewetting of a liquid film. Physical Review Fluids, 2016, 1, pp.41901(R). 10.1103/PhysRevFluids.1.041901 . cea-01485388

\section{HAL Id: cea-01485388 https://hal-cea.archives-ouvertes.fr/cea-01485388}

Submitted on 8 Mar 2017

HAL is a multi-disciplinary open access archive for the deposit and dissemination of scientific research documents, whether they are published or not. The documents may come from teaching and research institutions in France or abroad, or from public or private research centers.
L'archive ouverte pluridisciplinaire HAL, est destinée au dépôt et à la diffusion de documents scientifiques de niveau recherche, publiés ou non, émanant des établissements d'enseignement et de recherche français ou étrangers, des laboratoires publics ou privés. 


\title{
Evaporation-driven dewetting of a liquid film
}

\author{
L. Fourgeaud,,$^{1,2}$ E. Ercolani, ${ }^{3}$ J. Duplat,${ }^{3}$ P. Gully, ${ }^{3}$ and V. S. Nikolayev ${ }^{4, *}$ \\ ${ }^{1}$ PSA, Route de Gisy, 78140 Vélizy-Villacoublay, France \\ ${ }^{2}$ Université Grenoble Alpes, CEA, INAC, Service des Basses Températures, 38000 Grenoble, France \\ ${ }^{3}$ Service des Basses Températures, UMR-E CEA, UJF-Grenoble 1, INAC, 17 Rue des Martyrs, \\ 38054 Grenoble Cedex 9, France \\ ${ }^{4}$ Service de Physique de l'Etat Condensé, CEA, CNRS, Université Paris-Saclay, CEA Saclay, \\ 91191 Gif-sur-Yvette Cedex, France
}

(Received 13 January 2016; revised manuscript received 8 April 2016; published 16 August 2016)

We study the dynamics of evaporating ethanol films deposited by a receding liquid meniscus. The films are surrounded by pure vapor in a capillary heated above the saturation temperature. We observe the substrate dewetting with the dewetting ridge in spite of the complete wetting at equilibrium. The dewetting is caused by a high contact angle $\left(\sim 30^{\circ}\right)$ induced by evaporation. The obtained values agree with a theory proposed earlier. The film shape is measured with both grid deflection technique and interferometry. The phenomenon is convenient to observe inside a capillary with an axial thermal gradient. When the capillary is closed at one end and open at another to a constant pressure reservoir, the meniscus oscillations are known to appear spontaneously. Such a system is the simplest version of an industrial device called a pulsating heat pipe. The effect is general and can be used in any system to control the wetting properties.

DOI: 10.1103/PhysRevFluids.1.041901

Phase change (evaporation and condensation) occurring at the surface of thin liquid films is widely used as a means of intensive cooling of hot solid substrates. In any geometry, the substrate may become partially dry so that the gas-liquid-solid triple contact lines (CLs) form and move. The impact of evaporation or condensation on the CL motion has been studied theoretically for two limit cases. In the first limit case of strong evaporation, the phase change rate is controlled by the latent heat effect and heat exchange in the liquid, when it situates in the atmosphere of pure vapor of the same liquid [1-9]. This is the case, e.g., of bubbles in boiling. In the second limiting case, the phase change is controlled by the diffusion of vapor in the atmosphere of other gases [10-12]. This is the case, e.g., of a droplet drying in the open air. The phase change rate is generally much weaker than in the first case.

Unlike open air evaporation, in the pure vapor atmosphere case the Marangoni effects are unimportant (except at very high local heat fluxes where the kinetic interfacial resistance is important, e.g., very close to the contact line). The liquid-vapor interface is isothermal at saturation temperature $T_{\text {sat }}$ for the vapor pressure that is homogeneous. The latent heat plays a stabilizing role [13]. Once a local interface temperature fluctuation occurs, the phase change is induced and the accompanying latent heat effect compensates for the change. For example, if the interfacial temperature becomes higher, the evaporation occurs and the latent heat consumption leads to the temperature reduction until it becomes equal to $T_{\text {sat }}$ so that the evaporation stops.

For both limiting cases of evaporation, it has been shown that in the absence of the CL motion, the apparent contact angle $\theta_{\text {app }}$ is different from the contact angle with no evaporation $\theta$ and depends strongly on the phase change intensity. This difference appears because a high evaporation rate in the CL vicinity causes strong liquid flow towards it. The viscous pressure drop leads to the liquid surface bending causing the difference between the slope at the CL (equal to $\theta$ ) and at a microscopic

*Corresponding author: vadim.nikolayev@cea.fr 


\section{FOURGEAUD, ERCOLANI, DUPLAT, GULLY, AND NIKOLAYEV}
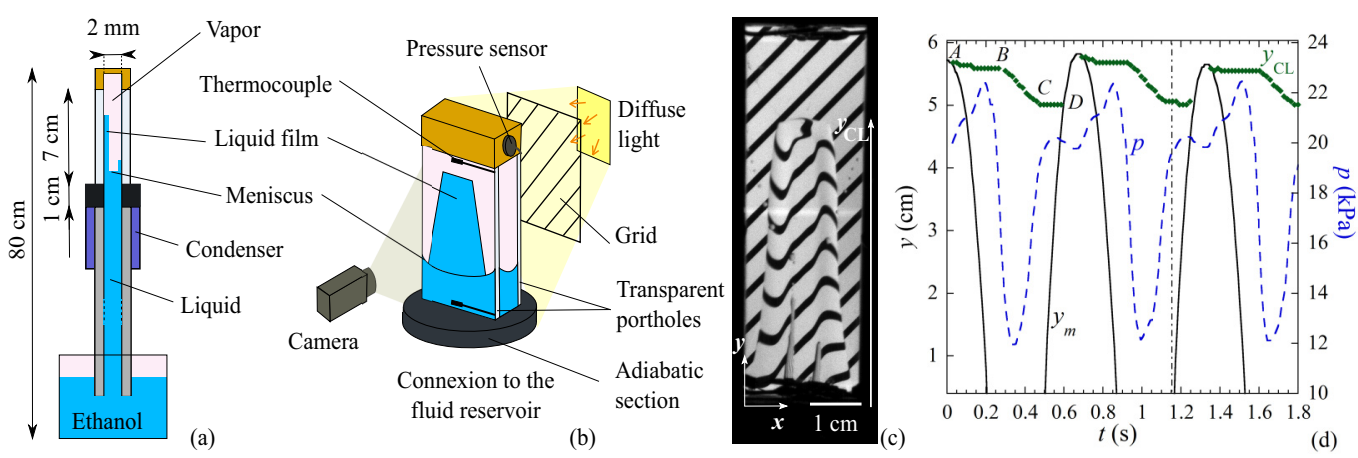

FIG. 1. (a) Schematic of the experiment. (b) Zoomed-in upper part with the optical deflectometry setup. (c) Raw camera image of the retracting trapezoidal film at the front porthole [in (b)]. Drops and rivulets remain from the rear film. Grid image discontinuity indicates the CL position; grid image distortion indicates the dewetting ridge. The coordinate system is also shown. (d) Time evolution of the meniscus position $y_{m}$, topmost $\mathrm{CL}$ position $y_{\mathrm{CL}}$, and vapor pressure $p$ measured for $p_{r}=23.7 \mathrm{kPa}, T_{c}=0{ }^{\circ} \mathrm{C}$, and heating powers 4 and $60 \mathrm{~W}$ at the front and rear portholes [in (b)], respectively. The oscillation frequency is $1.45 \mathrm{~Hz}$. The vertical dash-dotted line indicates the time of the image in (c) [18].

characteristic distance $l_{V}$ from it that corresponds to $\theta_{\text {app }}$. In the case of a pure vapor atmosphere, the evaporation rate is defined by the solid surface superheating $\Delta T=T_{s}-T_{\text {sat }}$, where $T_{s}$ is the solid surface temperature and $T_{\text {sat }}$ is the saturation temperature for the vapor pressure; $\theta_{\text {app }}$ is a function of $\Delta T$ only. This effect was found long ago [1] and was studied extensively since then by many researchers [2-5]. The dependence of $l_{V}$ on the phase change rate is weak, especially for the most common case where the hydrodynamic slip is relevant [9] and $l_{V}=3 l_{s} /(e \theta)$, where $l_{s}$ is the slip length and $e \approx 2.71$ is the Euler number [14].

In evaporation experiments, either the CL is pinned and $\theta_{\text {app }}$ is selected by the macroscopic meniscus shape [15] or CL moves and $\theta_{\text {app }}$ is influenced by the hydrodynamic flow related to the CL motion. The careful measurements of retraction of a sessile drop in the latent heat exchangecontrolled evaporation regime led to $\theta_{\text {app }}$ as a function of $\Delta T$ [16]. However, the influence of the CL motion on $\theta_{\text {app }}$ has been neglected. We account for it here. To describe the CL motion with the velocity $U_{\mathrm{CL}}$ (assumed positive at receding motion), one needs to consider, in addition to the microscopic region of the scale $l_{V}$, two more regions [14]: intermediate and macroscopic. The Cox-Voinov expression can be obtained by their asymptotic matching

$$
\theta_{\text {app }}^{3}=\theta_{V}^{3}-9 \mathrm{Ca}_{\mathrm{CL}} \ln \frac{W}{l_{V}},
$$

where $\mathrm{Ca}_{\mathrm{CL}}=\eta U_{\mathrm{CL}} / \gamma, \theta_{V}$ is the apparent contact angle caused by evaporation only (and discussed above), and $\theta_{V}=\theta_{\text {app }}\left(U_{\mathrm{CL}}=0\right)$. The macroscopic length $W$ should be defined by matching to the macroscopic region solution.

Because of scale separation, in the presence of evaporation, Eq. (1) suggests that the Voinov angle can be used universally instead of $\theta$. This is also supported by recent experimental observation of another manifestation of the $\theta_{V}>\theta$ phenomenon: evaporation-induced pinning on a microscopic substrate heterogeneity [17]. We present here macroscopic evidence: the evaporation-induced substrate dewetting by a fluid that however wets completely the substrate at equilibrium (which we verified with the ethanol drop spreading experiment).

The experimental system used for this purpose is the single-branch pulsating heat pipe [19]. It is a vertical capillary tube sealed from the top end and connected to a bottom reservoir maintained at a constant pressure $p_{r}$ [Fig. 1(a)] with a cooler and a regulated heater inside it. The capillary and reservoir form a closed vessel that contains a pure two-phase fluid. The middle part of the capillary is chilled at a constant temperature $T_{c}<T_{r} \equiv T_{\text {sat }}\left(p_{r}\right)$ and is called a condenser. The upper 


\section{EVAPORATION-DRIVEN DEWETTING OF A LIQUID FILM}

capillary part (evaporator) is heated and is partially filled with a vapor phase. The liquid meniscus can vertically oscillate around its equilibrium position. It is well known [20-23] that self-sustained oscillations can be induced in such a system due to alternating evaporation and condensation. During its receding, the meniscus deposits liquid film on the internal tube walls.

The film deposition without phase change (at isothermal conditions) has been described theoretically by Landau and Levich for the case of the flat plate pulled from a liquid pool [24] and later by Bretherton [25] for the case of a meniscus receding with the velocity $U_{m}$ in a capillary of the diameter $D$. The film thickness

$$
h_{0}=0.67 \mathrm{DCa}^{2 / 3}
$$

is defined by the capillary number $\mathrm{Ca}=\eta U_{m} / \gamma$, with $\eta$ the shear viscosity and $\gamma$ the surface tension. The deposited liquid film is unstable when the solid surface is not wettable. Once the CL is formed, it recedes spontaneously. For the flat plate case, the retracting film has a trapezoidal shape [26]. The liquid gathered by receding is accumulated in a dewetting ridge [27] formed at the film edge. When the ridge is small, its shape is controlled by the surface tension; its cross section is circular. When the ridge grows further, it transforms into a thick film $[26,28]$. Its thickness $\left[3 U_{\mathrm{CL}} \eta /(\rho g)\right]^{1 / 2}>h_{0}$ (with $g$ the gravity acceleration and $\rho$ the liquid density) is defined by the balance of viscous friction and gravity. In both cases (capillarity or gravity controlled ridge) $U_{\mathrm{CL}}$ is defined mainly by the static contact angle $\theta$ and thus is nearly independent of time. For the dewetting ridge geometry [29], $W=w / e$, where $w$ is the dewetting ridge width. As argued above, when phase change occurs at the contact line, the contact line motion is controlled by $\theta_{V}$ rather than by $\theta$, so the formula initially derived [29] for the isothermal case can be generalized as

$$
\mathrm{Ca}_{\mathrm{CL}}=\frac{\theta_{V}^{3}}{9}\left[\ln \left(a \mathrm{Ca}_{\mathrm{CL}}^{1 / 3} \frac{w^{2}}{l_{V} h}\right)\right]^{-1},
$$

where $a \approx 0.15$ and $h$ is the flat film thickness at the connection to the ridge $\left(h=h_{0}\right.$ for the isothermal case).

The cross section of the capillary used for the experiment described here is rectangular, $22 \times$ $2 \mathrm{~mm}^{2}$. The evaporator consists of two transparent sapphire portholes [Fig. 1(b)] fixed on the copper structure thus forming the Hele-Shaw cell. Indium tin oxide transparent layers are deposited at the outer surface of each porthole. They can be heated independently with an electric current. The evaporator is separated from the condenser by a 1-cm-high adiabatic section of capillary, made with a thermally insulating material (Vespel $\left.{ }^{\circledR}\right)$. Four thermocouples are glued on the portholes to control their temperature. The vapor pressure $p$ is measured by the fast response pressure transducer located at the top of the evaporator. The evaporator, which is the only transparent part of the tube, is filmed by a video camera. The device contains pure two-phase ethanol.

In the beginning of the experiment, the meniscus is brought into the evaporator by lowering $T_{c}$ (which leads to a decrease in $p$ ). When the evaporator heating is switched on, oscillations start. The oscillation dynamics can be described as follows [see Fig. 1(d)]. When the meniscus recedes from its topmost position $(t=0$, point $A$ ), it leaves the liquid films at both portholes. Their evaporation starts because $\Delta T>0$; vapor pressure $p$ rises. When the meniscus attains the condenser, the film condensation occurs at the lower film part [23]. In addition, the vapor expands. Both these effects cause a decrease in $p$ in spite of the continuing evaporation from the upper film part. The meniscus begins to rise under the action of the pressure difference $p_{r}-p$. Because of the liquid column inertia, the meniscus rises back into the evaporator in spite of the $p$ increase and the next oscillation occurs.

While receding, the meniscus deposits liquid films at the portholes. One of them is heated much more than the other (Fig. 1), so its film disappears completely during the first quarter of the period. The second quarter is then dedicated to measurements of the other film [18] while the meniscus is below the evaporator and thus out of the field of view. When the meniscus returns into the evaporator, it absorbs the films [point $D$ in Fig. 1(d)]. 
FOURGEAUD, ERCOLANI, DUPLAT, GULLY, AND NIKOLAYEV

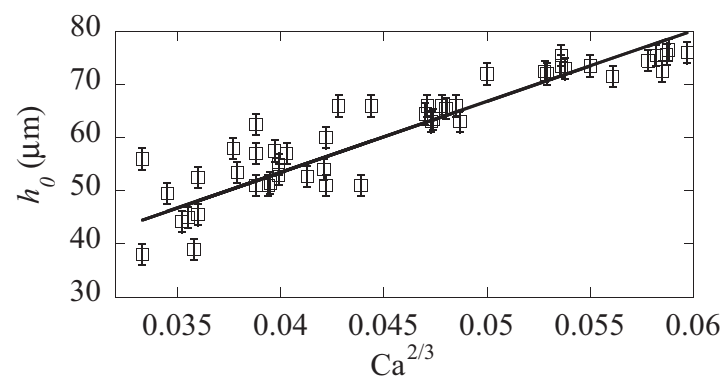

FIG. 2. Film thickness measured by interferometry (symbols) just after the film deposition at different heights to obtain different meniscus velocities. It is compared to Eq. (2) (line, calculated with $D=2 \mathrm{~mm}$ corresponding to the cell thickness; $\gamma=22.3 \times 10^{-3} \mathrm{~N} / \mathrm{m}$, and $\eta=7.9 \times 10^{-4} \mathrm{Pas}$ ).

To determine the contact angle evolution, it is necessary to reconstruct the whole film shape. Recently, two vain attempts [30,31] to measure the dynamic profile of the evaporating liquid film were reported, which shows both the importance and difficulty of this task.

The film thickness $h$ as a function of the vertical $(y)$ and horizontal $(x)$ coordinates is measured using two nonintrusive optical techniques. The first is the grid deflection technique [26,32,33]. A wire grid is placed between the diffuse light source and the cell [Fig. 1(b)]. The wire direction is inclined to $45^{\circ}$ to avoid it to be parallel to the CL. The incident light rays are deflected at the inclined film interface. The local film slope can be deduced from distortion of the grid image [Fig. 1(c)]. A local $h$ measurement is needed to reconstruct its spatial variation along the single wire image. This measurement is provided by the interferometry technique as described below.

Figure 1(d) shows two regimes. When $p$ is smaller than some value (17 $\mathrm{kPa}$ in this case) so that $\Delta T$ is large and evaporation is strong, the CL recedes (between points $B$ and $C$ ); it stays immobile between points $C$ and $D$. The grid image distortion at the CL (corresponding to a large $\theta_{\text {app }}$ ) is low when the pressure is high [18]; the distortion nearly disappears between points $C$ and $D$, indicating $\theta_{\text {app }}$ reduction.

The trapezoidal film shape closely resembles that observed in the study of isothermal LandauLevich films in the case of partial wetting [26]. The grid image is not distorted in the central film part. This means that it is nearly flat and its thickness can be measured by interferometry at a fixed height similarly to [28]. The film thickness (Fig. 2) measured right after meniscus passage in front of the interferometer is relatively well described by Eq. (2) in spite of the inertial distortion of the meniscus curvature that changes the prefactor of the equation. Different points in Fig. 2 have been obtained in the same run by performing the simultaneous $h_{0}$ and $U_{m}$ measurements at different heights.

The thickness has been measured by interferometry at nine regularly spaced heights in the central film part during the same experimental run. Due to the periodic (with good accuracy) motion, the data can be reduced to a single period as if they were taken simultaneously at different heights to obtain the time evolution of the film profile (Fig. 3). The film thins right after its deposition and a tiny $\left(\sim 0.06^{\circ}\right)$ film inclination can be seen. It originates from evaporation. For the film deposited earlier, the thickness decrease caused by evaporation is stronger, so a film slope appears [31]. For this reason and because of the complete wetting of sapphire by ethanol at equilibrium, one could expect a priori the wedgelike film profile. The film shape reconstruction using the grid deflection technique (Fig. 4) reveals, however, a different picture, similar to the isothermal dewetting at partial wetting conditions [26]: The central film part is linked to a ridge through a capillary jump visible in the inset in Fig. 3, where the ridge temporal evolution is shown. The ridge shape is well fitted by circular arcs except at late times [roughly, between points $C$ and $D$ in Fig. 1(d)], where the gravity distorts its profile initiating a transition to the thick film [28] regime.

The three-dimensional reconstruction and a good quality of the circular fits of the lateral ridges allow the temporal variation of $\theta_{\text {app }}$ to be defined with sufficient precision [Fig. 5(a)]. To obtain $\theta_{V}$, 


\section{EVAPORATION-DRIVEN DEWETTING OF A LIQUID FILM}

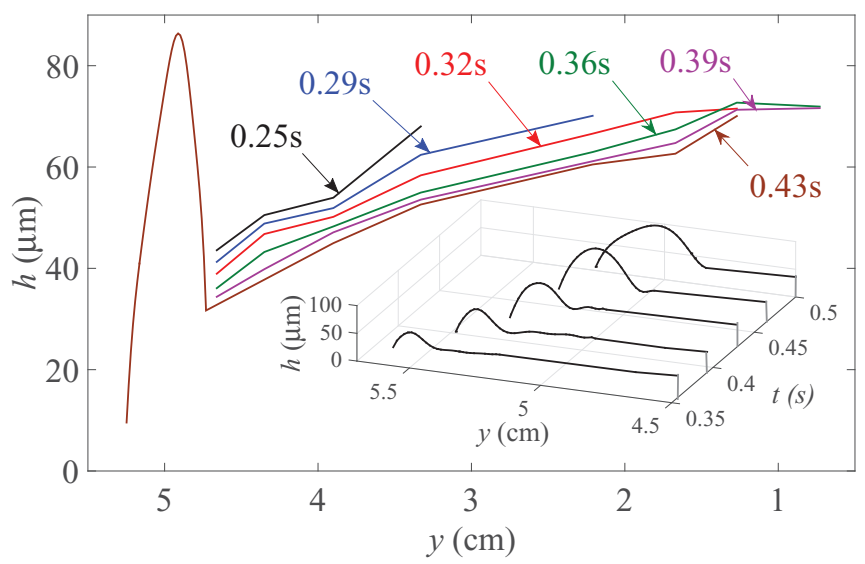

FIG. 3. Time evolution of the central film part. The film slope is smaller than $0.01^{\circ}$ (note the scale difference of the axes $y$ and $h$ ). For one profile, the upper ridge is shown schematically to indicate its relative size and position. The inset shows the time evolution of the upper ridge profile determined with the grid deflection technique.

the CL velocity term needs to be accounted for in Eq. (1). By taking $w \sim 3 \mathrm{~mm}$ and $l_{s} \sim 20 \mathrm{~nm}$ [34] (which results in $l_{V} \sim 100 \mathrm{~nm}$ ), the logarithmic value is $\sim 9$ and $\theta_{V}$ can now be calculated [Fig. 5(a)]. High $\theta_{V}$ values contrast with complete wetting at equilibrium and appear due to strong evaporation in the CL vicinity as described theoretically above. Indeed, the $\theta_{V}$ increase is correlated with the $T_{\text {sat }}$ drop below a threshold. The $\theta_{V}$ increase causes the sapphire dewetting so that CL depins from the surface defects and recedes, $U_{\mathrm{CL}}$ grows, and the capillary ridge forms. When $T_{\text {sat }}$ increases again, $\theta_{V}$ drops to a small value. The CL becomes pinned again and $w$ grows [18].

Because of the porthole thermal inertia, the internal surface temperature $T_{s}$ in the contact line vicinity (which is, unfortunately, impossible to be measured directly) does not vary strongly during oscillations. On the external surfaces, the temperature is weakly oscillating $\left( \pm 1^{\circ} \mathrm{C}\right)$ and varies spatially between $37^{\circ} \mathrm{C}$ at the bottom of the front porthole [that with the film; see Fig. 1(b)], and $57^{\circ} \mathrm{C}$ at the top of the rear porthole, to which a higher heating power is applied. By neglecting the effect of fluid flow along the film, one can obtain its thickness decrease rate

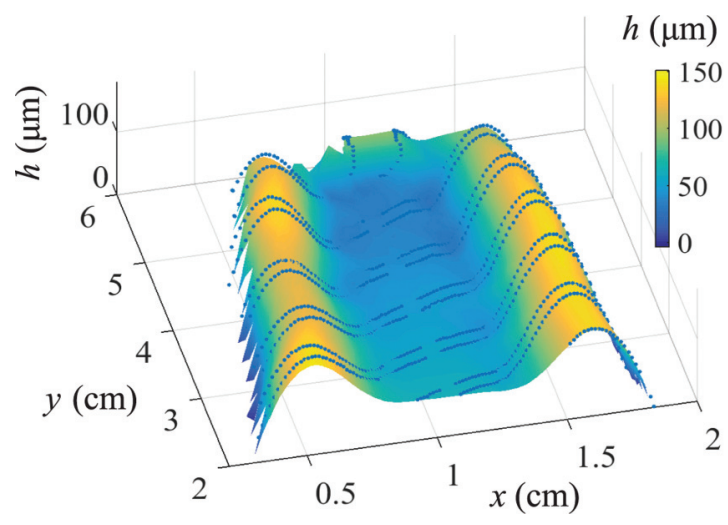

FIG. 4. Three-dimensional reconstruction of the film profile corresponding to the image in Fig. 1(c). The surface is obtained by interpolation between reconstructed points (symbols) along the images of grid edges; $\theta_{\text {app }}=7 \pm 1^{\circ}$. 
FOURGEAUD, ERCOLANI, DUPLAT, GULLY, AND NIKOLAYEV

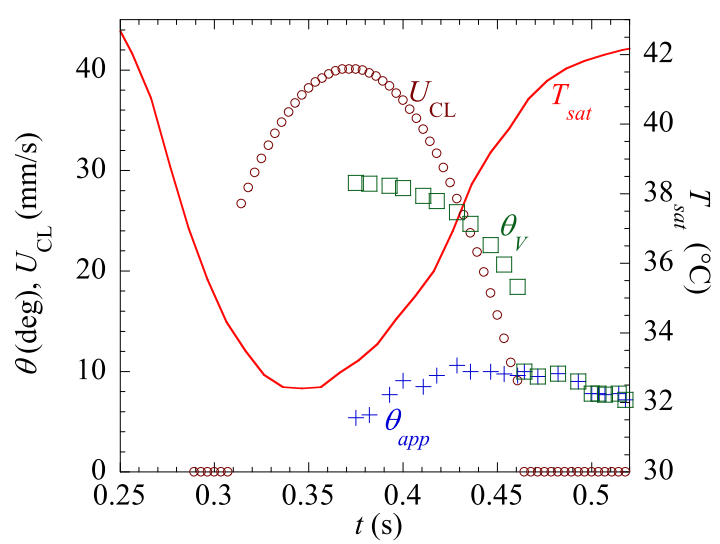

(a)

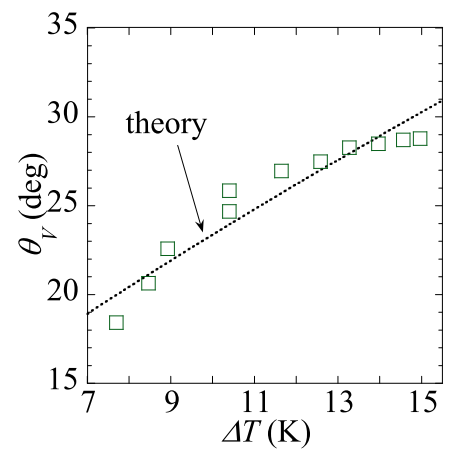

(b)

FIG. 5. (a) Plot of $\theta_{V}$ (squares) and $T_{\text {sat }}$ (solid line) [cf. $p(t)$ shown in Fig. 1(d)] temporal variations. Also shown are $U_{\mathrm{CL}}$ (circles) and $\theta_{\text {app }}$ (crosses), both measured at the right inclined portion of the CL at $y=3 \mathrm{~cm}$ height. The precision for $\theta$ is $\pm 2^{\circ}$. (b) Plot of $\theta_{V}$ versus $\Delta T=T_{s}-T_{\text {sat }}$. The symbols denote $\theta_{V}$ and $T_{\text {sat }}$ data extracted from the graph (a) for the moving (depinned) $\mathrm{CL} ; T_{s}=48^{\circ} \mathrm{C}$ is assumed. The dotted line is the theoretical curve calculated for ethanol with the approach described in Ref. [9].

$\partial h / \partial t=-J / \rho$ during the film residence time after deposition, where $J=\lambda\left(T_{s}-T_{\mathrm{sat}}\right) /(h L)$ (with $L$ the latent heat and $\lambda$ the heat conductivity). The resulting equation can be solved for $h(t)$ with $T_{\text {sat }}(t)$ known from Fig. 5(a) and a constant $T_{s}$. By fitting the experimental evolution of $h(t)$ (Fig. 3), one obtains the estimation $T_{s}=48^{\circ} \mathrm{C}$. With this value, one can plot $\theta_{V}(t)$ versus $\Delta T(t)$ for the sake of comparison with the theory [9] [see Fig. 5(b)]. The agreement is good. Only the $\theta_{V}$ values selected by evaporation (i.e., for the depinned CL) should be compared.

Once the $\theta_{V}$ evolution is known, one can determine the contact line receding velocity from Eq. (3) as if it were defined only by the capillary effect. It is plotted in Fig. 6 together with the experimental contact line velocity. One can see that the experimental velocity is slightly larger, by about $14 \%$. This deviation should result from the evaporative mass loss at the contact line, i.e., evaporation in the contact line vicinity $[9,16]$.

The impact of evaporation was considered for the liquid film deposition by the receding meniscus at complete wetting conditions. One would expect a wedgelike film shape because the film deposited earlier evaporates longer and should thus become thinner. The observed shape is however different:

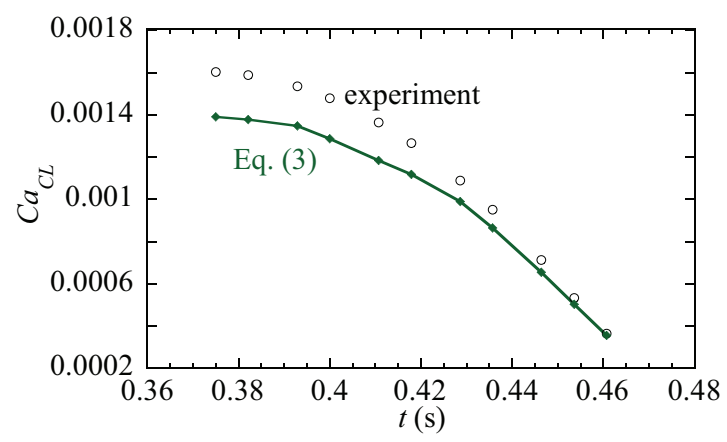

FIG. 6. Dimensionless contact line receding velocity. The theoretical curve is the capillary dewetting velocity calculated with Eq. (3) using the $\theta_{V}(t)$ and $w(t)$ data from Figs. 5(a) and 3, respectively. The experimental values (circles) correspond to the $U_{\mathrm{CL}}$ data of Fig. 5(a). 


\section{EVAPORATION-DRIVEN DEWETTING OF A LIQUID FILM}

The film slope is tiny $\left(<0.1^{\circ}\right)$ and a ridge is formed at its edge. The ridge is explained by the dewetting phenomenon appearing because of the high contact angle created at the microscopic level due to the strong microscale evaporation at the contact line. The value found $\left(\simeq 30^{\circ}\right)$ agrees with a theoretical model developed earlier. The contact line receding velocity is mainly controlled by the capillary dewetting; the contribution of the liquid mass loss at the contact line is responsible only for $14 \%$ of the velocity. These results will be useful to control the wetting properties with evaporation or condensation and to understand many industrially important phenomena such as boiling or the functioning of an industrial device called a pulsating heat pipe (the experimental installation presents its simplest version).

We thank A. Gauthier for his technical assistance throughout this project. We are grateful to B. Andreotti for his help and advice concerning the film thickness measurements and to D. Garcia and F. Bancel for their help. The financial contributions of Agence Nationale de la Recherche within the project AARDECO ANR-12-VPTT-005-02 and of ESA within MAP INWIP are acknowledged.

[1] M. Potash and P. C. Wayner, Evaporation from a two-dimensional extended meniscus, Int. J. Heat Mass Transfer 15, 1851 (1972).

[2] S. Moosman and G. M. Homsy, Evaporating menisci of wetting fluids, J. Colloid Interface Sci. 73, 212 (1980).

[3] D. M. Anderson and S. H. Davis, The spreading of volatile liquid droplets on heated surfaces, Phys. Fluids 7, 248 (1995).

[4] L. M. Hocking, On contact angles in evaporating liquids, Phys. Fluids 7, 2950 (1995).

[5] S. J. S. Morris, Contact angles for evaporating liquids predicted and compared with existing experiments, J. Fluid Mech. 432, 1 (2001).

[6] V. Janeček and V. S. Nikolayev, Contact line singularity at partial wetting during evaporation driven by substrate heating, Europhys. Lett. 100, 14003 (2012).

[7] A. Rednikov and P. Colinet, Singularity-free description of moving contact lines for volatile liquids, Phys. Rev. E 87, 010401 (2013).

[8] V. Janeček, B. Andreotti, D. Pražák, T. Bárta, and V. S. Nikolayev, Moving contact line of a volatile fluid, Phys. Rev. E 88, 060404 (2013).

[9] V. Janeček and V. S. Nikolayev, Apparent-contact-angle model at partial wetting and evaporation: Impact of surface forces, Phys. Rev. E 87, 012404 (2013).

[10] C. Poulard, O. Bénichou, and A. M. Cazabat, Freely receding evaporating droplets, Langmuir 19, 8828 (2003).

[11] J. Eggers and L. M. Pismen, Nonlocal description of evaporating drops, Phys. Fluids 22, 112101 (2010).

[12] V. V. Janeček, F. Doumenc, B. Guerrier, and V. S. Nikolayev, Can hydrodynamic contact line paradox be solved by evaporation-condensation? J. Colloid Interface Sci. 460, 329 (2015).

[13] Y. Garrabos, C. Lecoutre-Chabot, J. Hegseth, V. S. Nikolayev, D. Beysens, and J.-P. Delville, Gas spreading on a heated wall wetted by liquid, Phys. Rev. E 64, 051602 (2001).

[14] J. H. Snoeijer and B. Andreotti, Moving contact lines: Scales, regimes, and dynamical transitions, Annu. Rev. Fluid Mech. 45, 269 (2013).

[15] H. Hu and R. G. Larson, Evaporation of a sessile droplet on a substrate, J. Phys. Chem. B 106, 1334 (2002).

[16] R. Raj, C. Kunkelmann, P. Stephan, J. Plawsky, and J. Kim, Contact line behavior for a highly wetting fluid under superheated conditions, Int. J. Heat Mass Transfer 55, 2664 (2012).

[17] Y. Tsoumpas, S. Dehaeck, M. Galvagno, A. Rednikov, H. Ottevaere, U. Thiele, and P. Colinet, Nonequilibrium Gibbs criterion for completely wetting volatile liquids, Langmuir 30, 11847 (2014). 


\section{FOURGEAUD, ERCOLANI, DUPLAT, GULLY, AND NIKOLAYEV}

[18] See Supplemental Material at http://link.aps.org/supplemental/10.1103/PhysRevFluids.1.041901 for a video.

[19] S. P. Das, V. S. Nikolayev, F. Lefèvre, B. Pottier, S. Khandekar, and J. Bonjour, Thermally induced two-phase oscillating flow inside a capillary tube, Int. J. Heat Mass Transfer 53, 3905 (2010).

[20] M. Rao, F. Lefèvre, S. Khandekar, and J. Bonjour, Understanding transport mechanism of a self-sustained thermally driven oscillating two-phase system in a capillary tube, Int. J. Heat Mass Transfer 65, 451 (2013).

[21] V. S. Nikolayev, Oscillatory instability of the gas-liquid meniscus in a capillary under the imposed temperature difference, Int. J. Heat Mass Transfer 64, 313 (2013).

[22] M. Rao, F. Lefèvre, S. Khandekar, and J. Bonjour, Heat and mass transfer mechanisms of a self-sustained thermally driven oscillating liquid-vapour meniscus, Int. J. Heat Mass Transfer 86, 519 (2015).

[23] V. S. Nikolayev, Effect of tube heat conduction on the single branch pulsating heat pipe start-up, Int. J. Heat Mass Transfer 95, 477 (2016).

[24] L. D. Landau and B. V. Levich, Dragging of a liquid by a moving plate, Acta Physicochim. URSS 17, 42 (1942).

[25] F. P. Bretherton, The motion of long bubbles in tubes, J. Fluid Mech. 10, 166 (1961).

[26] J. H. Snoeijer, G. Delon, M. Fermigier, and B. Andreotti, Avoided Critical Behavior in Dynamically Forced Wetting, Phys. Rev. Lett. 96, 174504 (2006).

[27] F. Brochard-Wyart, J.-M. de Meglio, and D. Quéré, Démouillage. Etude du retrait d'un film de liquide non mouillant déposé sur un plan ou une fibre, C. R. Acad. Sci. II 304, 553 (1987).

[28] J. H. Snoeijer, J. Ziegler, B. Andreotti, M. Fermigier, and J. Eggers, Thick Films of Viscous Fluid Coating a Plate Withdrawn from a Liquid Reservoir, Phys. Rev. Lett. 100, 244502 (2008).

[29] J. H. Snoeijer and J. Eggers, Asymptotic analysis of the dewetting rim, Phys. Rev. E 82, 056314 (2010).

[30] V. Srinivasan, V. Marty-Jourjon, S. Khandekar, F. Lefèvre, and J. Bonjour, Evaporation of an isolated liquid plug moving inside a capillary tube, Int. J. Heat Mass Transfer 89, 176 (2015).

[31] N. Chauris, V. Ayel, Y. Bertin, and C. Romestant, Evaporation of a liquid film deposited on a capillary heated tube: Experimental analysis by infrared thermography of its thermal footprint, Int. J. Heat Mass Transfer 86, 492 (2015).

[32] V. Gurfein, D. Beysens, Y. Garrabos, and B. Le Neindre, Simple grid technique to measure refractive index gradients, Opt. Commun. 85, 147 (1991).

[33] J. Hegseth, A. Oprisan, Y. Garrabos, V. S. Nikolayev, C. Lecoutre-Chabot, and D. Beysens, Wetting film dynamics during evaporation under weightlessness in a near-critical fluid, Phys. Rev. E 72, 031602 (2005).

[34] E. Lauga, M. P. Brenner, and H. A. Stone, in Springer Handbook of Experimental Fluid Dynamics, edited by C. Tropea, A. Yarin, and J. Foss (Springer, New York, 2007), Chap. 19, pp. 1217-1240. 